\title{
Improving Social Support to Increase QoL in Lung Cancer Patients
}

This article was published in the following Dove Press journal:

Cancer Management and Research

\author{
Adriana Hofman (D) \\ Natalia Zajdel (D' \\ Jakub Klekowski $\mathbb{D}^{\prime}$ \\ Mariusz Chabowski (iD) 2,3 \\ 'Student Research Group No 180, \\ Faculty of Medicine, Wroclaw Medical \\ University, Wrocław, Poland; ${ }^{2}$ Division of \\ Oncology and Palliative Care, \\ Department of Clinical Nursing, Faculty \\ of Health Science, Wrocław Medical \\ University, Wrocław, Poland; \\ ${ }^{3}$ Department of Surgery, 4th Military \\ Teaching Hospital, Wrocław, Poland
}

Correspondence: Mariusz Chabowski Department of Surgery, 4th Military Teaching Hospital, 5 Weigla Street, Wroclaw, 50-98I, Poland

Tel +48 26I 660247

$\mathrm{Fax}+48261660245$

Email mariusz.chabowski@gmail.com

\begin{abstract}
Lung cancer is the most common cause of cancer deaths in the world. According to the World Health Organization, over 2 million new lung cancer cases were reported worldwide in 2018, and there were 1.76 million deaths from the disease. Due to its nonspecific symptoms, the disease is usually detected at an advanced stage, which gives few treatment options and a short survival rate after diagnosis. That is why improving QoL in the last months of a patient's life is enormously important. The purpose of this study was to analyse original papers in order to determine whether an increase in social support is important in improving QoL for lung cancer patients. Both the direct influence of social support on QoL and indirect influences such as stigma or depression, etc. were taken into consideration. We conducted a review of 22 papers published in English in the period 2005-2020, which were selected using the following keywords: lung cancer, social support, quality of life. Evidence was found for the connections between the improvement of social support and an increase in the QoL of lung cancer patients. It is crucial, and worth whatever effort is required, to increase social support for lung cancer patients, as it is one of the most important factors in improving QoL. According to the studies, a good QoL and minimalised symptoms of the disease are much more important for lung cancer patients than the length of their life.
\end{abstract}

Keywords: quality of life, lung cancer, social support, stigma, depression

\section{Introduction}

Lung cancer is the most common cause of cancer deaths in the world. According to the World Health Organization (WHO), over 2 million new lung cancer cases were reported worldwide in 2018, and there were 1.76 million deaths from the disease. In Europe, there were more than 500,000 new cases in 2018 and over 435,000 people died of lung cancer. It is estimated that over 541,000 people lived with a diagnosis of lung cancer in the US in 2015 . $^{1}$ The 5-year survival rate in Europe is $19 \%$, and this is one of the lowest results among other most common types of cancer. ${ }^{2}$ Due to its non-specific symptoms, the disease is usually detected at an advanced stage, which gives few treatment options. Patients are usually advanced in years, and many of them have metastases located far away from the primary tumour, so curative treatment is possible in fewer than $20 \%$ of cases. ${ }^{2}$ Treatment options for lung cancer include surgery, chemotherapy, and radiation therapy, all of which are very burdensome, especially for elderly patients. Due to the short survival rate after diagnosis and many other special factors associated with this type of cancer, there is a need to maintain the best possible quality of life in lung cancer patients. 


\section{Quality of Life}

Quality of life (QoL) is a broad concept, defined by many authors in different ways. According to the WHO, it is an individual's perception of someone's position in life in the context of the culture and value systems in which someone lives and in relation to someone's goals, expectations, standards and concerns.

It is believed that the assessment of QoL in patients diagnosed with lung cancer is currently gaining in importance as a complement to the patient's diagnosis and can be an important predictive factor. ${ }^{3,4}$ It allows the detection of disease-related limitations and needs which are identified by the patient, but which are not necessarily included in general medical and therapeutic management. ${ }^{4}$ Moreover, it can comprehensively evaluate the effectiveness of the anticancer ruleset. ${ }^{5}$ The QoL assessment includes physical aspects (physical condition, difficulties in performing daily activities, presence of symptoms of the disease), mental aspects (emotions related to the disease, anxiety, depression), ability to handle cognitive processes (memory, attention) and social contacts, as well as the ability to carry out life roles (work, hobbies). ${ }^{4}$

Patients' QoL is influenced by multiple factors, such as: gender, age, the intensity of the symptoms of the disease and the therapy, the number of symptoms (such as: fatigue, pain, insomnia, cough, dyspnea, nausea and vomiting, weight loss), the type of treatment, the histological cancer type, and the stage of the disease; the patient's mental health, motivation and attitude, their level of education, history of smoking, socioeconomic status, marital status, social, economic and emotional support, and their employment status (Table 1).

In comparison with other cancers, QoL in lung cancer patients was significantly worse. $^{3-7}$ Moreover, Pongthavornkamol et al mentioned that the pain was much more intense in patients with lung cancer than in patients with other cancers, which may be due to late diagnosis of lung cancer and the numerous symptoms present in later stages of the disease. Given the statistically short survival time of lung cancer patients and the toxic and not always effective treatment they receive, priority should be given to assessing QoL and improving QoL in this group of patients. ${ }^{5,8}$

\section{Social Support}

Social support consists of social functions and relationships. A distinction is made most often between perceived
Table I Factors Influencing the Quality of Life (QoL) in Lung Cancer Patients

\begin{tabular}{|c|c|}
\hline Factors Raising QoL & Factors Lowering QoL \\
\hline $\operatorname{Men}^{4,6}$ & Women ${ }^{4,29}$ \\
\hline Under 65 years $^{4}$ & Above 65 years ${ }^{4,24}$ \\
\hline Fewer symptoms ${ }^{4}$ & More symptoms ${ }^{4,24}$ \\
\hline Milder physical symptoms ${ }^{4}$ & Severe physical symptoms ${ }^{4,6,8,24}$ \\
\hline Early palliative care ${ }^{4}$ & Pain and fatigue $e^{4,6,7,28}$ \\
\hline Surgical treatment ${ }^{4,29}$ & Chemotherapy, ${ }^{4,29}$ metastases ${ }^{5,29,37}$ \\
\hline $\begin{array}{l}\text { Non-Small Cell Lung Cancer } \\
(\mathrm{NSCLC})^{5}\end{array}$ & Small Cell Lung Cancer (SCLC) ${ }^{4,5}$ \\
\hline Non-smoker ${ }^{4}$ & Smoker ${ }^{4}$ \\
\hline Spirituality ${ }^{6,28}$ & Stigma ${ }^{39,40}$ \\
\hline High level of education ${ }^{6}$ & Low level of education ${ }^{29}$ \\
\hline $\begin{array}{l}\text { Social security, economic } \\
\text { support, }^{15} \text { employed or } \\
\text { retired }^{4,6}\end{array}$ & Low income, ${ }^{4,5,29}$ unemployed $d^{4,6}$ \\
\hline $\begin{array}{l}\text { High perceived social } \\
\text { support } 3,6,17,28\end{array}$ & Low perceived social support ${ }^{3,5}$ \\
\hline Married or widowed ${ }^{15,29,30}$ & Unmarried ${ }^{5,29}$ or divorced ${ }^{30}$ \\
\hline High coping skills ${ }^{4,17}$ & Poor coping skills ${ }^{4}$ \\
\hline $\begin{array}{l}\text { High self-efficacy }{ }^{4,24} \text { and high } \\
\text { cognitive abilities }{ }^{4}\end{array}$ & $\begin{array}{l}\text { Indifference and } \\
\text { inactivity, }{ }^{4} \text { hopelessness }{ }^{29}\end{array}$ \\
\hline Functional independence ${ }^{6,28}$ & Low compliance ${ }^{4 !}$ \\
\hline $\begin{array}{l}\text { Less intensive anxiety or } \\
\text { depression }{ }^{4}\end{array}$ & Depression, anxiety $4,5,29,37$ \\
\hline
\end{tabular}

support and the support that is actually received. The first relates to anticipating the patient's needs, and the second to the actual help provided over time. The difference is significant, since these two constructs need not necessarily have much in common. Social support is an interactive process and can be related to altruism, a sense of obligation and the perception of reciprocity. Research has shown that social support can be expressed in several ways, including instrumental support (eg physical/medical assistance), material support (eg financial), informational support (eg advising, educating) and emotional support (eg empathy). ${ }^{9}$ Support can come from a variety of sources such as partners, family, friends, healthcare professionals and other patients. ${ }^{10}$ Material support can be provided by 
the government, foundations, family, insurance companies, donors and others. Patients in difficult life situations and with socioeconomic burdens have a greater need of support. Therefore, healthcare providers should identify a patient's needs and provide as much support as that patient requires. ${ }^{11}$ Due to the characteristics of the disease and its treatment, lung cancer patients need a particularly high level of support and therefore it is very important to develop effective supportive care interventions for these patients. $^{12}$ Social support has been proven to have a positive impact on the health, mental and cognitive wellbeing and quality of life of the patient and his/her caregiver. ${ }^{13-15}$ In addition, social support is a significant factor in protecting against negative mental states, reducing unpredictable behaviour and mortality. ${ }^{16-20}$

Social support influences quality of life through two psychological mechanisms: behavioural processes (eg prohealth behaviour, adherence) and psychological processes (stress appraisal). ${ }^{21}$ Early implementation of support measures improves the median survival of patients even in the metastatic stage. ${ }^{22}$

\section{Materials and Methods}

We conducted the search for articles in the PubMed, EBSCO Information Services, Web of Science, UpToDate, Ovid and Scopus electronic databases using the following key words: social support, lung cancer, and quality of life. The inclusion criteria were: English language and year of publication between January 2005 and August 2020; and the direct or indirect impact of social support on quality of life in patients with lung cancer. The initial selection enabled us to extract 115 articles. We excluded: papers unrelated to lung cancer patients, and those not describing direct or indirect impact of social support on quality of life in patients, and reviews. The final analysis comprised 22 papers.

\section{Results}

In a Korean multicenter study of 1956 cancer patients by Eom et al, several important and interesting results were obtained. Conclusions indicating a significant positive direct relationship between social support and quality of life was the most essential factor, as were results associating low QoL in patients with low perceived social support. The authors investigated additional correlations to social support and concluded that low perceived social support results in higher levels of depression, higher scores regarding symptoms (fatigue, nausea, vomiting, pain, dyspnea, sleep disturbance, constipation, diarrhea) and financial problems. Interestingly, patients living in cities tend to have lower levels of perceived social support compared to those living in rural areas. The researchers proved that group treatment focusing on the complicated reactions to grief improves perceived social support, which was furthermore demonstrated to ameliorate the severity of depression. The authors concluded that their studies have important implications because of the beneficial impact of social support on QoL, both for clinicians and public health, and they suggested that early intervention aimed at improving social support should be introduced immediately after the diagnosis, being either hospital or community-based, and focusing on those with low base social support. $^{23}$

Regarding Applebaum et al's study, higher levels of perceived social support were significantly associated with better QoL in 168 patients from United States (US) with various types of advanced cancer. According to this study, social support is an important factor affecting QoL and resilience. Social support was reported to be a protective factor against morbidity, mortality, distress, psychopathology, and to promote psychological adjustment to chronic diseases. Interestingly, it was also found that for patients with high levels of optimism who experience social support on a daily basis, additional social support may be a signal of decreasing health status, as family and friends traditionally increase their support as the physical wellbeing of cancer patients' declines. ${ }^{3}$

Liao et al found that personalised supportive care, especially information provision, self-efficacy skill training, symptom management, and counselling, was crucial for improving the QoL in patients with advanced lung cancer. In addition, they indicated that patients with newly diagnosed advanced lung cancer experience more psychosocial distress than physical distress. They concluded that current methods improved the physical aspects of lung cancer treatment and the availability of information, but psychosocial health assessment and counseling still required development. ${ }^{24}$

A study of 350 US patients with newly diagnosed incurable lung or noncolorectal gastrointestinal cancer demonstrated that emotional support and acceptance coping strategies were associated with better QoL and mood. $^{17}$

$\mathrm{Gu}$ et al's study of 148 patients in China found that social support is particularly important in the clinical management of lung cancer, because it was plausible to 
observe that patients with poor social support had a lower level of psychological and physical QoL. ${ }^{5}$

In the study by Bertero et al, the researchers conducted a study in which 23 patients took part. The participants were interviewed about their life experience and quality of life after being diagnosed with lung cancer. Later on, the data was assessed using a phenomenological hermeneutic approach. The researchers stated that the essence of their study was "living as usual". This led to the conclusion that the patients with lung cancer valued their independence and that not being able to manage by themselves greatly decreased their quality of life. The participants mentioned that they had problems asking for help from members of their support network, as it could make them feel dependent. Therefore, the authors concluded that the quality of life of patients with lung cancer can be enhanced by improving social support in order to help them cope with uncertainty in their new and difficult life situation. Additionally, this article also drew attention to promoting support for the next of kin of patients, as they were often an important aspect of patients' QoL experience. ${ }^{25}$

A study by Huang et al, based on 103 participants, showed the prevalence of functional weakness and supportive care needs in patients with advanced lung cancer. People with greater need for external help, and especially psychological support, experienced a lower QoL than more independent patients. The authors suggested that early identification of those requiring particular support, which could be noted during the assessment by physicians, and the implementation of this support may increase QoL in patients with advanced lung cancer. ${ }^{26}$

Raz et al indicated that early implementation of social support improves QoL and survival in patients with metastatic lung cancer. In their study, which was carried out on 71 patients, they found a clinically significant improvement of QoL and levels of distress in patients 12 months after lung cancer surgery, due to the implementation of interdisciplinary supportive care. ${ }^{20}$

Lee and Jeong thoroughly investigated factors contributing to QoL of NSCLC patients. They studied 212 lung cancer patients, finding that QoL was directly influenced by symptom burden and resilience, while distress and social support had an indirect impact on QoL. The authors noticed an indirect pathway of social support to QoL through resilience and the correlation was a positive one. Among the four predictors that were studied, distress had the most significant impact on QoL, while resilience and social support influenced the levels of distress. ${ }^{27}$ The findings of this study implied not only the importance of social support to QoL through resilience, but perhaps even more importantly they showed the influence of social support on other factors that lower QoL in lung cancer patients.

In John's study, patients reported that having family and friends nearby improved their QoL. They concluded that social support was very important in maintaining QoL. Moreover, patients reported problems with the availability of support groups for lung cancer patients and the feeling of stigma while participating in a support group with people affected by other types of cancer. ${ }^{28}$

A study by Boehmer et al covered 175 gastrointestinal, colorectal and lung cancer patients. In this study, the social support that was received was discovered to only have a significantly positive influence on emotional well-being (one of three health-related QoL domains), whereas it had almost zero significant correlation with social and physical wellbeing. ${ }^{10}$ This was the only study we found that showed almost no relationship between social support and QoL. However, the conclusions should be noted with caution as the study did not relate only to lung cancer patients.

\section{Marital Status}

Aizer et al carried out research on 734,889 patients with many types of cancers - including lung cancer. They point out the significant impact that social support received from a spouse has on cancer treatment and survival. They propose intervening with social support as a solid part of care offered to unmarried patients diagnosed with cancer, which they claim would have a positive impact on the improvement of treatment outcomes in these patients. ${ }^{22}$ Similarly, Gu et al and Jatoi et al found that unmarried patients had a poorer QoL than married patients. ${ }^{29,30}$ Moreover, several other studies concluded that the level of perceived social support in unmarried patients was lower than in married patients. ${ }^{23,30,31}$ In our view, these outcomes may have a significant correlation and indicate that the support received from a relative has a positive impact on QoL. In Germany, 173 couples in which one person was suffering from lung cancer were examined by Luszczynska et al. They found out that women with cancer received less support from their partner than male patients from their spouses. Luszczynska also mentioned that emotional support seemed more important than any other kind of support. ${ }^{32}$ 


\section{Depression}

Depression is a common, yet often overlooked and not well understood problem for patients with lung cancer. ${ }^{17}$ Research shows that among patients suffering from lung cancer between $21-44 \%$ are depressed, $49 \%$ are anxious and $51 \%$ are distressed - according to the HADS classification scores. ${ }^{33-35}$ The risk factors for developing depression after diagnosis were identified as patient characteristics, a family history of depression, less social support, poor communication with medical caregivers and maladaptive coping strategies. Current smoking status has been also brought up as a risk factor for new onset depression during lung cancer treatment. ${ }^{36}$ One study suggests that developing depression may be due to the side effects of ongoing lung cancer treatment. ${ }^{17}$ Gonzalez and Jacobsen found a significant link between the previously mentioned stigma and symptoms of depression. ${ }^{35}$ Important conclusions come from some studies which show a significantly lower quality of life in lung cancer patients additionally suffering from depression. ${ }^{36,37}$ Moreover, there are findings suggesting a worse survival rate in lung cancer patients who developed depression, especially those with low levels of social support. ${ }^{36}$

A study of 216 Turkish lung cancer patients revealed a negative relationship between depression, hopelessness and perceived social support. It is worth mentioning that patients scored high in perceived social support from family, although the levels of perceived social support from family decreased the longer the disease continued. ${ }^{38}$ Sullivan et al studied 1790 patients and found that more cancer symptoms and less social support were associated with higher mortality among lung cancer patients suffering from depression. They concluded that providing adequate social support should be a key component of any depression management programme as part of comprehensive lung cancer care. ${ }^{36}$

Feeling depressed and being younger were pointed out as factors decreasing QoL in women suffering from lung cancer in the study by Sarna et al. The patients also reported that cancer impacted their sexual functioning and levels of distress in the family, which affected their social well-being. ${ }^{37}$ Khue et al emphasised that the treatment of the disease should not be limited to treating the etiology of the condition. It should also include the search for the psychological effects of the disease. They said that depression and anxiety can affect a patient's quality of life and that this was often neglected. These symptoms could be treated effectively at a low cost. ${ }^{21}$

The multitude of symptoms occurring in patients with lung cancer had a negative impact on their physical condition, and anxiety and helplessness further reduced the quality of their mental health. Gu et al mentioned that social support is helpful in reducing stress and adapting to dealing with the disease. ${ }^{29}$

It is important to take all these findings into account when planning treatment schemes for lung cancer patient and to also consider depression screening in this group.

\section{Smoking and Stigma}

Smoking is estimated to be responsible for $80 \%$ of all deaths from lung cancer in the US. ${ }^{39}$ Patients suffering from lung cancer are often linked with guilt and punishment resulting from tobacco use. ${ }^{37}$ As a result of this, $30-95 \%$ of patients with lung cancer feel stigmatised because of their disease, irrespective of whether they are smokers or non-smokers. ${ }^{39}$ The relationship between lung cancer and smoking, accented by years of anti-tobacco campaigns, has strengthened the belief that lung cancer patients are in some way responsible for their diagnosis and this leads to an internalisation of blame. ${ }^{18}$ Johnson et al claimed that stigma may be important in terms of QoL for lung cancer patients and may independently influence social support and physical symptoms. ${ }^{39}$ Chambers et al also noted that patients who feel this stigma face shame, fear and rejection, and have more limited social networks, all of which lead to diminished QoL and lower psychological welfare. $^{33}$ It is important to provide increased social support to these patients in order to reverse the impact of stigma, as it leads to social isolation, intensified distress, unsupportive relationships and resentfulness towards the patient. ${ }^{18,33}$ However, the sources of social support that are easily available to patients, such as support groups, are often scarce. In one study, the lung cancer patients who were interviewed noticed problems finding any support groups specifically for lung cancer patients, and while visiting other general cancers support groups, they encountered a smoker-shaming approach from other participants. ${ }^{28}$

An important correlation between QoL and social support influenced by sigma was found in the study by Johnson et al. The research was based on 62 participants with advanced stage lung cancer. Statistical evidence showed less social support in patients with high stigma. Furthermore, the article pointed out that stigma negatively 
influenced the QoL of the patients. Thus, the authors concluded that social support may influence the general experience of lung cancer patients in the final months of life. ${ }^{39}$ Chambers et al confirmed their study's hypothesis and found that health-related stigma and psychological distress were responsible for poorer QoL in lung cancer patients. Based on that finding, the authors recommend increasing efforts aimed at fighting health-related stigma in order to improve QoL and that those endeavours should bear in mind the social sources of stigma. Additionally, an acceptance-focused psychological intervention was conducted in this study, which resulted in lower stigma among lung cancer patients. ${ }^{33}$

\section{Discussion}

Many of the studies we analysed confirmed that social support had a significant positive influence on QoL in patients with lung cancer. We strongly believe that increasing social support will result in an improved quality of life for patients with lung cancer.

This correlation can be applied globally, as the studies we analysed were conducted with patients not only from Europe, but also from the USA, Vietnam, China and India, among others.

Social support received from family, relatives, and other patients with the same disease, as well as from medical staff and the community influences the patients' quality of life, both directly and indirectly, by means of lessening the impact of factors which reduce QoL, such as depression, anxiety and stigmatisation.

Patients often indicated the need for their relatives to be present during a hospital stay or medical consultation, which should be respected and enabled as much as possible. ${ }^{11}$ Cultivating supporters' commitment to medical appointments often improved the treatment experience, especially in patient-physician communication. ${ }^{14}$

Patients with lung cancer are a specific group of patients; they struggle with the high level of stigma, they are subjected to, have a higher level of depression and anxiety, and have a lower QoL than patients with other cancers, as well as having a short survival time after diagnosis together with sustained and very burdensome treatment. Many of these factors can be positively influenced by increasing social support and it is definitely worth doing, so that in the last months or years of life this will result in better treatment results, as QoL is one of the components of treatment outcome. Moreover, increasing QoL in these patients does not require a large financial outlay, since depression can be treated at a low cost pharmacologically $^{21}$ or in support groups which, however, are currently not sufficiently accessible to patients with lung cancer. ${ }^{28}$

Anti-smoking campaigns are a major source of the aversion and rejection directed towards lung cancer patients. Posters and slogans that show the negative effects of smoking and the disease that can result from a patient's deliberate and conscious behaviour, and the stigmatising reaction of the public, cause the patient to experience a deep feeling of guilt and lack of acceptance by others. ${ }^{18}$

There are some groups of patients who have insufficient support or have higher support needs, such as women, patients with a low economic status, and those who are unmarried. ${ }^{23,30-32}$ Several researchers say that it is extremely important to identify these patients and to provide them with more support. To this end, it is necessary to systematically assess social support and QoL and respond to any deficiencies that emerge in people from these groups in order to improve patients' QoL. ${ }^{23,24,26,39}$

Providing social support is important not only for patients with lung cancer, but also for their caregivers, who are the most important in supporting and sustaining a patient confronting the disease. Kelley et al examined 218 lung and 222 colorectal cancer patients and found out that social support has a positive impact on the health of both the patient and their caregiver. The results of their research suggested that having more social support, especially of the emotional and informational kind, helped to encourage a reduction in the number of risk behaviours. ${ }^{18}$

Many studies showed that financial (material) support was also extremely important for increasing patients' wellbeing, as the costs of treatment, supplements, and rehabilitation that they need to cover were a huge burden. ${ }^{14,21}$ Undoubtedly, the financial burden caused by the disease was one of the most important problems faced by patients and reported in the research. Patients in a better financial position coped better with their recovery. ${ }^{16}$ People in a difficult economic situation had higher levels of depression and anxiety. Moreover, reduced income and job loss resulted in a lower QoL. ${ }^{40}$ Nayak et al found that $93.6 \%$ of the 768 patients suffering from various cancers who took part in their study in India reported below-average economic well-being. ${ }^{8}$

Although social support was initially perceived to be good by patients with lung cancer, there were several ways to make it even better. ${ }^{41}$ We believe that the support offered to patients should be multidimensional, coming 
synchronously from different sources and tailored to each patient individually. ${ }^{11,16,24}$

In many cases the diagnosis itself caused shock, disorientation and a sense of helplessness. Patients are exposed to a completely new, unknown situation. It is important that in such situations the medical personnel inform the patient about ways of coping with the disease as well as the existing support groups, offering the patient the opportunity to participate in them. In studies we analysed, the patients indicated a lack of access to support groups, while there is a huge need for them to exist and to be available. Perhaps it would be worthwhile setting up such groups within specialist units dealing with patients with lung cancer. Uchitomi et al concluded that support from a physician had a significant impact on a patient's fighting spirit. ${ }^{42}$ We believe that the outcome of this study should have a practical importance and this relationship should be used in order to encourage patients to increase their efforts in fighting the disease.

As the studies showed, patients had a significant need for up-to-date information about their health and treatment results and many of them complained about a lack of informational support. ${ }^{11,34}$ The accessibility of medical staff throughout the entire disease was very important in order for the patient to perceive that they were receiving comprehensive care and for them to feel as safe and comfortable as possible.

As many patients were interested in exercises and activities that would improve the efficiency of their respiratory system, it would be worthwhile to instruct and teach them to improve their self-efficacy and abilities. ${ }^{11}$ A very important element of patient care and improving well-being was also the promotion of physical activity and the motivation for patients to change their lifestyle. In our opinion, it may be helpful if not only the doctor mobilises the patient, but also support groups, rehabilitation programmes, special phone applications, and online training videos. Such motivational support results in a comprehensive and a substantial increase in QoL. ${ }^{43}$ Missel et al discovered that the social benefits of group exercise were enormous and made the patient feel that they belonged to a certain community. Exercises and meetings with people who had had the same experiences were very important for them, as they made it easier to tolerate the illness and therapy, as well as creating an atmosphere of respect and minimalised fear. ${ }^{44}$

Due to the increase in the number of oncological patients, the limited time available to medical staff and the high cost of their work, we should seriously consider the development of social support for example on the web. ${ }^{45}$ Currently, the majority of people use smartphones, which undoubtedly offers a huge opportunity for the promotion of applications or programmes for community gatherings, rehabilitation and exercise programmes, teleconsultation and education about cancer, treatment and health improvement. Thanks to such procedures, patients feel cared for, have a sense of belonging to a community, are motivated to exercise, can freely communicate with people in a similar situation and benefit from interpersonal contacts and quick access to reliable advice and news in case of doubt, without waiting in a queue to see the doctor. $^{44,46}$ Sui et al conducted a study where patients who had undergone surgical NSCLC resection used the We-Chat app-based education and rehabilitation program (WERP) for 12 months, and found that the application significantly improved patients' QoL and well-being. The phone application WERP is based on disease-related health education, rehabilitation exercise guidance, daily activity supervision and psychological support. Patients who used the application had lower levels of anxiety and depression. Researchers concluded that WERP could be an effective way of reducing anxiety and depression and improving QoL in patients after NSCLC surgery. ${ }^{46} \mathrm{We}$ believe that in these times of the COVID-19 pandemic, when the availability of medical services is particularly limited, the utilisation of online support methods is especially valuable and worthwhile.

Since there are few original studies available that describe the direct impact of social support on QoL in lung cancer patients, and these studies are often based on different scales and tests, it is difficult to compare them precisely and to draw conclusions. Therefore, we have tried to take into consideration here the influence of various factors such as stigma, depression or marital status on QoL in lung cancer patients and the influence of social support on these factors. On this basis, we have allowed ourselves to reach conclusions about the indirect impact of these factors on QoL in lung cancer patients. We presume that the unification of social support and QoL assessment methods could help future researchers draw conclusions more precisely and facilitate the comparison of subsequent studies. Although the standardisation of the scales mentioned above is desirable, we conclude that the hypothesis of our study has been confirmed and that social support is of substantial importance in increasing QoL in lung cancer patients. 


\section{Funding}

The authors received no financial support for this research and the authorship of this article.

\section{Disclosure}

The authors report no conflicts of interest in this work.

\section{References}

1. Noone AM, Howlader N, Krapcho M, et al. SEER cancer statistics review, 1975-2015. Bethesda, MD: National Cancer Institute. Based on November 2017 SEER data submission, posted to the SEER web site; April, 2018. Available from: https://seer.cancer.gov/csr/1975_ 2015/. Accessed March 1, 2021.

2. American Cancer Society. Cancer Facts \& Figures 2020. Atlanta: American Cancer Society; 2020.

3. Applebaum AJ, Stein EM, Lord BJ, Pessin H, Rosenfeld B, Breitbart W. Optimism, social support, and mental health outcomes in patients with advanced cancer. Psycho-Oncology. 2014;23 (3):299-306. doi:10.1002/pon.3418

4. Polanski J, Jankowska-Polanska B, Rosinczuk J, Chabowski M, Szymanska-Chabowska A. Quality of life of patients with lung cancer. Onco Targets Ther. 2016;2016(Issue 1):1023-1028.

5. Gu W, Xu YM, Zhong BL. Health-related quality of life in Chinese inpatients with lung cancer treated in large general hospitals: acrosssectional study. BMJ Open. 2018;8(4):e019873. doi:10.1136/bmjopen-2017-019873

6. Hung $\mathrm{H}, \mathrm{Wu} \mathrm{L}$, Chen K. Determinants of quality of life in lung cancer patients. J Nurs Scholarsh. 2018;50(3):257-264. doi:10.1111/ jnu. 12376

7. Pongthavornkamol K, Lekdamrongkul P, Pinsuntorn P, Molassiotis A. Physical symptoms, unmet needs, and quality of life in Thai cancer survivors after the completion of primary treatment. Asia Pac J Oncol Nurs. 2019;6(4):363-371. doi:10.4103/apjon. apjon_26_19

8. Nayak M, George A, Shashidhara Y, Nayak B. Symptom interference and relation between the domains of quality of life among cancer patients of tertiary care hospital. Indian J Palliat Care. 2019;25 (4):575-579. doi:10.4103/IJPC.IJPC_139_19

9. Schwarzer R, Knoll N, Rieckmann N. Social support. In: Kaptein A, Weinman J, editors. Health Psychology. Malden, UK: Blackwell; 2004:158-181.

10. Boehmer S, Luszczynska A, Schwarzer R. Coping and quality of life after tumor surgery: personal and social resources promote different domains of quality of life. Anxiety Stress Coping. 2007;20(1):61-75. doi:10.1080/10615800701195439

11. Zhang T, He H, Liu Q, Lv X, Song Y, Hong J. Supportive care needs of patients with lung cancer in Mainland China: a cross-sectional study. J Nurs Res. 2019;27(6):e52. doi:10.1097/jnr.0000000000000338

12. Porter LS, Keefe FJ, Garst J, et al. Caregiver-assisted coping skills training for lung cancer: results of a randomized clinical trial. J Pain Symptom Manage. 2011;41(1):1-13. doi:10.1016/j.jpainsymman. 2010.04.014

13. Huang FF, Yang Q, Zhang J, Han XY, Zhang JP. The structural equation model on self-efficacy during post-op rehabilitation among non-small cell lung cancer patients. PLoS One. 2018;13(9):e0204213. doi:10.1371/journal.pone.0204213

14. Ellis KR, Black KZ, Baker S, et al. Racial differences in the influence of health care system factors on informal support for cancer care among black and white breast and lung cancer survivors. Fam Community Health. 2020;43(3):200-212. doi:10.1097/FCH.000 0000000000264
15. Dogan N, Tan M. Quality of life and social support in patients with lung cancer. Int J Caring Sci. 2019;12(1):263-269.

16. Hu T, Xiao J, Peng J, Kuang X, He B. Relationship between resilience, social support as well as anxiety/depression of lung cancer patients: a cross-sectional observation study. J Cancer Res Ther. 2018;14(1):72-77. doi:10.4103/jcrt.JCRT_849_17

17. Nipp RD, El JA, Fishbein JN, et al. The relationship between coping strategies, quality of life, and mood in patients with incurable cancer. Cancer. 2016;122(13):2110-2116. doi:10.1002/cncr.30025

18. Kelley DE, Kent EE, Litzelman K, Mollica MA, Rowland JH. Dyadic associations between perceived social support and cancer patient and caregiver health: an actor-partner interdependence modeling approach. Psychooncology. 2019;28(7):1453-1460. doi:10.1002/pon.5096

19. Pinquart M, Duberstein PR. Associations of social networks with cancer mortality: a meta-analysis. Crit Rev Oncol Hematol. 2010;75 (2):122-137. doi:10.1016/j.critrevonc.2009.06.003

20. Raz DJ, Sun V, Kim JY, et al. Long-term effect of an interdisciplinary supportive care intervention for lung cancer survivors after surgical procedures. Ann Thorac Surg. 2016;101(2):495-503. doi:10.1016/j. athoracsur.2015.07.031

21. Khue PM, Thom VT, Minh DQ, Quang LM, Hoa NL. Depression and anxiety as key factors associated with quality of life among lung cancer patients in Hai Phong, Vietnam. Front Psychiatry. 2019;10. doi:10.3389/fpsyt.2019.00352.

22. Aizer AA, Chen M-H, McCarthy EP, et al. Marital status and survival in patients with cancer. J Clin Oncol. 2013;31(31):3869-3876. doi:10.1200/JCO.2013.49.6489

23. Eom C, Shin DW, Kim SY, et al. Impact of perceived social support on the mental health and health-related quality of life in cancer patients: results from a nationwide, multicenter survey in South Korea. Psycho-Oncology. 2013;22(6):1283-1290. doi:10.1002/ pon.3133

24. Liao Y-C, Shun S-C, Liao W-Y, Yu C-J, Yang P-C, Lai Y-H. Quality of life and related factors in patients with newly diagnosed advanced lung cancer: a longitudinal study. Oncol Nurs Forum. 2014;41(2): E44-E55. doi:10.1188/14.ONF.E44-E55

25. Berterö C, Vanhanen M, Appelin G. Receiving a diagnosis of inoperable lung cancer: patients' perspectives of how it affects their life situation and quality of life. Acta Oncol. 2008;47(5):862-869. doi:10.1080/02841860701654333

26. Huang ZP, Cheng HL, Loh SY, Cheng KKF. Functional status, supportive care needs, and health-related quality of life in advanced lung cancer patients aged 50 and older. Asia Pac J Oncol Nurs. 2020;7(2):151-160. doi:10.4103/apjon.apjon_50_19

27. Lee JL, Jeong Y. Quality of life in patients with non-small cell lung cancer: structural equation modeling. Cancer Nurs. 2019;42 (6):475-483. doi:10.1097/NCC.0000000000000645

28. John LD. Self-care strategies used by patients with lung cancer to promote quality of life. Oncol Nurs Forum. 2010;37(3):339-347. doi:10.1188/10.ONF.339-347

29. Gu W, Xu YM, Zhong B-L. Health-related quality of life in Chinese inpatients with lung cancer treated in large general hospitals: a cross-sectional study. BMJ Open. 2018;8(4):1. doi:10.1136/bmjopen-2017-019873

30. Jatoi A, Novotny P, Cassivi S, et al. Does marital status impact survival and quality of life in patients with non-small cell lung cancer? Observations from the mayo clinic lung cancer cohort. Oncologist. 2007;12(12):1456-1463. doi:10.1634/theoncologist.12$12-1456$

31. Aydın Sayılan A, Demir Doğan M. Illness perception, perceived social support and quality of life in patients with diagnosis of cancer. Eur $J$ Cancer Care (Engl). 2020;29(4):1-7. doi:10.1111/ecc.13252

32. Luszczynska A, Boehmer S, Knoll N, Schulz U, Schwarzer R. Emotional support for men and women with cancer: do patients receive what their partners provide? Int J Behav Med. 2007;14 (3):156-163. doi:10.1007/BF03000187 
33. Chambers SK, Baade P, Youl P, et al. Psychological distress and quality of life in lung cancer: the role of health-related stigma, illness appraisals and social constraints. Psycho-Oncology. 2015;24 (11):1569-1577. doi:10.1002/pon.3829

34. Liao YC, Liao WY, Shun SC, et al. Symptoms, psychological distress, and supportive care needs in lung cancer patients. Support Care Cancer. 2011;19(11):1743-1751. doi:10.1007/s00520-010-1014-7

35. Gonzalez BD, Jacobsen PB. Depression in lung cancer patients: the role of perceived stigma. Psychooncology. 2012;21(3):239-246. doi: $10.1002 /$ pon. 1882

36. Sullivan DR, Forsberg CW, Ganzini L, et al. Depression symptom trends and health domains among lung cancer patients in the CanCORS study. Lung Cancer. 2016;100:102-109. doi:10.1016/j. lungcan.2016.08.008

37. Sarna L, Brown JK, Cooley ME, et al. Quality of life and meaning of illness of women with lung cancer. Oncol Nurs Forum. 2005;32:9-19. doi:10.1188/05.ONF.E9-E19

38. Şahin ZA, Tan M, Polat H. Hopelessness, depression and social support with end of life Turkish cancer patients. Asian Pac J Cancer Prev. 2013;14(5):2823-2828. doi:10.7314/ APJCP.2013.14.5.2823

39. Johnson LA, Schreier AM, Swanson M, Moye JP, Ridner S. Stigma and quality of life in patients with advanced lung cancer. Oncol Nurs Forum. 2019;46(3):318-328. doi:10.1188/19.ONF.318-328

40. Akin S, Can G, Aydiner A, Ozdilli K, Durna Z. Quality of life, symptom experience and distress of lung cancer patients undergoing chemotherapy. Eur J Oncol Nurs. 2010;14(5):400-409. doi:10.1016/ j.ejon.2010.01.003
41. Jie Z, Yizhen Y, Anni W, et al. Resilience in patients with lung cancer: structural equation modeling. Cancer Nurs. 2020. doi:10.1097/NCC.0000000000000838

42. Uchitomi Y, Mikami I, Kugaya A, et al. Physician support and patient psychologic responses after surgery for nonsmall cell lung carcinoma: a prospective observational study. Cancer. 2001;92 (7):1926-1935. doi:10.1002/1097-0142(20011001)92:7<1926::AIDCNCR1711>3.0.CO;2-L

43. Clark MM, Novotny PJ, Patten CA, et al. Motivational readiness for physical activity and quality of life in long-term lung cancer survivors. Lung Cancer. 2008;61(1):117-122. doi:10.1016/j. lungcan.2007.12.012

44. Missel M, Borregaard B, Schoenau MN, Sommer MS. A sense of understanding and belonging when life is at stake-Operable lung cancer patients' lived experiences of participation in exercise. Eur $J$ Cancer Care. 2019;28(5):e13126. doi:10.1111/ecc.13126

45. Yao T, Zheng Q, Fan X. The impact of online social support on patients' quality of life and the moderating role of social exclusion. J Serv Res. 2015;18(3):369-383. doi:10.1177/1094670515583271

46. Sui Y, Wang T, Wang X. The impact of WeChat app-based education and rehabilitation program on anxiety, depression, quality of life, loss of follow-up and survival in non-small cell lung cancer patients who underwent surgical resection. Eur J Oncol Nurs. 2020;45. doi:10.1016/j.ejon.2019.101707.

\section{Publish your work in this journal}

Cancer Management and Research is an international, peer-reviewed open access journal focusing on cancer research and the optimal use of preventative and integrated treatment interventions to achieve improved outcomes, enhanced survival and quality of life for the cancer patient.
The manuscript management system is completely online and includes a very quick and fair peer-review system, which is all easy to use. Visit http://www.dovepress.com/testimonials.php to read real quotes from published authors. 